\title{
Graphene and MWCNT: Potential Candidate for Microwave Absorbing Materials
}

\author{
Pallab Bhattacharya \\ Materials Science Centre, Indian Institute of Technology \\ Kharagpur 721302, West Bengal, India \\ Tel: 91-964-122-7589Ｅ-mail: pallabcm@gmail.com \\ Chapal Kumar Das (Corresponding author) \\ Materials Science Centre, Indian Institute of Technology \\ Kharagpur 721302, West Bengal, India \\ Tel: 91-322-028-3978_E-mail: chapal12@yahoo.co.in
}

Swinderjeet Singh Kalra

Department of Chemistry, D. A-V College

Kanpur 208001, India

Tel: 91-983-903-9952Ｅ-mail: sskdav@rediffmail.com

Received: February 2, 2012

doi:10.5539/jmsr.v1n2p126
Accepted: February 16, 2012 Published: Apri1 1, 2012

URL: http://dx.doi.org/10.5539/jmsr.v1n2p126

\begin{abstract}
Currently a wide range of materials are used for the design and development of microwave absorbing material or RADAR absorbing materials (RAMs). The microwave has two components, electric and magnetic which are acting perpendicular to each other. So, in order to make materials microwave invisible, it is required to cancel out both of these components, when material is exposed to microwave i.e. reduction of radar cross section (RCS). The RAMs should capable of cancelling out both the magnetic and electrical components of the electromagnetic radiation for an effective absorption. Generally this has been achieved by incorporating magnetic and electrically conducting fillers into a matrix. But here we want to study the microwave absorption ability of Graphene/MWCNT itself in TPU matrix. We prepared the material with $10 \%$ loading and sample thickness kept at $2 \mathrm{~mm}$. Field Emission Scanning electron microscopy (FESEM) and Transmission Electron Microscopy (TEM) used for morphological study and scattering parameters were measured in X-band region by using a Vector Network Analyzer. Result showed that Graphene has better absorption capability than MWCNT.
\end{abstract}

Keywords: Graphene, MWCNT, Composite, Radar absorbing material, Microwave absorption

\section{Introduction}

Usage of RADAR absorbing materials (RAMs) or Microwave absorbing materials for commercial and military application is been started since 1930s (Das, et al., 2011). But still it is a topic of great interest in research field. One of the materials extensively studied as RADAR absorbing materials is carbon nanotube (CNT). CNT is a promising candidate for this application due to its properties like high conductivity, small diameter, high aspect ratio and super mechanical strength. That is why the investigations of electro-dynamic properties of composites based on different polymer matrices and CNTs are very popular topics in materials science (Wang, et al., 2005; Fan, et al., 2006). Recently, CNTs with epoxy (Li, et al., 2006; Matzui, et al., 2007), polystyrene (PS), polyaniline (PANI) (Wang, et al., 2005; Watts, et al., 2003; Ma, et al., 2005), polymethylmethacrylate (PMMA) (Wang, et al., 2005; Fan, et al., 2006), polypropylene (Liu, et al., 2007; Al-Saleh, et al., 2009), etc., have been studied for the possible applications as effective and lightweight RAMs. It is also reported that defective multi-walled carbon nanotubes (MWCNTs) have high permittivity between 8-18 GHz (Watts, et al., 2003). Recently Graphene is another candidate on this context because it has also special structures and properties. So 
the reports on Graphene and CNT for microwave application lead us to study their individual microwave absorption ability in X-Band region (8.2-12.4 GHz). Graphene has flat sheet-like structure whereas CNT has tubular structure. We thought that may be due to their structural variety they will act in a different way in the microwave region. According to the reference (Hao, et al., 2008) plate-shaped material has an advantage in effective absorption over the rod-like or sphere-shaped material. Hence we studied here the microwave absorption ability of two composite based on Graphene and MWCNT in thermoplastic polyurethane (TPU) matrix. We prepared those composites and their morphological study was studied by Field Emission Scanning electron microscopy (FESEM) and Transmission electron microscopy (TEM) and its microwave characteristics was studied by Agilent vector network analyzer (ENA E5071C). Best of our knowledge it is still not reported elsewhere. The matrix used for the preparation of different RAMs is thermoplastic polyurethane (TPU). Total filler percentage was maintained around $10 \%$ and the thicknesses of the RAMs were $2 \mathrm{~mm}$.

\section{Experimental Work}

\subsection{Materials}

Graphene was obtained from Graphene Supermarket, Calverton, NY. MWCNT was purchased from Reinste Nano Ventures Pvt. Ltd., India. Tetrahydrofuran $\left(\mathrm{C}_{4} \mathrm{H}_{8} \mathrm{O}\right)$ was purchased from Merck, India. The TPU utilized for developing RAMs belong to commercial medical grade aliphatic, polyether (TecoflexVR EG 80A injection grade) procured from Lubrizol Advanced Materials, Thermedics TM Inc. Polymer Products, USA. Tecoflex EG $80 \mathrm{~A}$ (around $35 \%$ of hard segments) has shore hardness of $72 \mathrm{~A}$, specific gravity $=1.04$, and its constituent formulation contains methylene bis (cyclohexyl) diisocyanate (HMDI) as hard segment, and polytetramethylene oxide (PTMO) as soft segment (molecular weight $=1000 \mathrm{~g} / \mathrm{mol}$ ), and chain extender 1, 4-butane di-ol (BD).

\subsection{Modification of Graphene and MWCNT}

The surface modification of Graphene was carried out using mixed acid $\left(\mathrm{HNO}_{3} / \mathrm{H}_{2} \mathrm{SO}_{4}\right)$. At first Graphene was treated with a solution of $\mathrm{H}_{2} \mathrm{SO}_{4}$ and $\mathrm{HNO}_{3}$ (weight ratio of $\mathrm{H}_{2} \mathrm{SO}_{4}$ to $\mathrm{HNO}_{3}$ was 3:1). Here the weight ratio of the mixed acid to Graphene was 200:1. Then the whole solution was stirred at $60^{\circ} \mathrm{C}$ for 24 hours to get acid modified Graphene. After that the solution was centrifuged and washed with distilled water and ethanol for several times. Then the prepared black coloured solution was dried at $70^{\circ} \mathrm{C}$ for 12 hours. In the same procedure MWCNT was also modified prior to use in further composite preparation.

\subsection{Preparation of Composite}

To disperse the modified Graphene and MWCNT in TPU matrices, at first TPU was dissolved in $200 \mathrm{ml}$ THF at $60^{\circ} \mathrm{C}$ by stirring it for overnight and then cooled it at room temperature before the addition of the fillers. After well dispersion of the fillers, solvent was evaporated to make the mixture highly concentrated. Then this concentrated mixture was poured on a petri dish and kept in oven at $70^{\circ} \mathrm{C}$ for complete drying. After drying the test samples were prepared by compression molding technique at $5 \mathrm{MPa}$ pressure and $170^{\circ} \mathrm{C}$ temperature. Aluminum sheet was used as metallic backing material of the developed RAMs. Samples were prepared with $10 \%$ loading with Graphene and MWCNT. Thickness of the RAM was maintained to $2 \mathrm{~mm}$. Both the samples were cut into desired rectangular shape of size 0.4 inch $\times 0.9$ inch to fit into X-band waveguide for microwave measurements.

\section{Characterization}

The high resolution microstructural details were achieved by the Field Emission Scanning electron microscopy (FESEM) study carried out on Carl Zeiss-SUPRA 40. Transmission electron microscopy (TEM) was obtained on a JEOL JEM-2100 microscope. The microwave absorption study was done by using Agilent vector network analyzer (ENA E5071C).

\subsection{Results and Discussion}

\subsection{Morphological Study}

\subsubsection{FESEM Analysis}

Figure 1 showed the FESEM images for the fractured cross-section, generated by cryogenic treatment, of Graphene and MWCNT in presence of TPU. Graphene has sheet like structure and MWCNT showing its tubular shape in the FESEM images. Dispersion of Graphene and MWCNT was confirmed by their FESEM images.

\subsubsection{TEM Analysis}

For further morphological study of the composite, we have done TEM analysis and presented in the Figure 2. In TEM study tubular structure of MWCNT is more prominent this proved the presence of MWCNT in the composite. 


\subsection{Microwave Absorbing Properties}

From the scattering parameters $S_{11} \& S_{21}$, we have calculated the reflection coefficient $(\tau)$ with the help of thefollowing equations (Das, et al., 2012) -

$$
\tau=x \pm \sqrt{\left(x^{2}-1\right)} ;|\tau| \leq 1
$$

Where $x=\left(\mathrm{S}^{2}{ }_{11}-\mathrm{S}_{21}^{2}+1\right) / 2 \mathrm{~S}_{11}$

Reflection loss $($ in $\mathrm{dB})=-20 \log [\tau]$

Figure 3 represented the return loss Vs frequency plot for Graphene and MWCNT present in TPU matrix. The prepared RAMs showed the absorbing properties in a wide frequency range in the X-band region. Graphene and MWCNT in TPU showed the maximum return loss of $-12.56 \mathrm{~dB}$ at $10.43 \mathrm{GHz}$ and $-7.6 \mathrm{~dB}$ at $10.73 \mathrm{GHz}$ respectively. Both were showing their maximum return loss peak almost in the same frequency range but Graphene showed better absorption capacity than MWCNT. According to transmission line theory, (Michielssen, et al., 1993) when the electromagnetic wave transmits through a medium, the reflectivity is affected by many factors such as permittivity, permeability, sample thickness, and electromagnetic wave frequency. Here the frequency of radiation and thickness of sample is same for all samples. So to explain the difference in absorption ability of the RAMs we studied their permittivity and permeability characteristics.

\subsection{Relative Complex Permittivity and Permeability}

According to the theory of permittivity, when a material comes under the influence of an electromagnetic field, the electric field induces two types of electrical currents within the material, i.e., conduction and displacement current. The former arises due to the presence of free electrons for conduction and gives imaginary part of permittivity $\left(\mathrm{e}^{\prime \prime}\right)$. The latter arises due to the bound charges, i.e., polarization and gives real part of permittivity (e') (Anju Gupta, et al., 2011).To investigate the possible mechanism of microwave absorption, we determined the real and imaginary parts of complex permittivity $\left(\mathrm{e}^{\prime}, \mathrm{e}^{\prime \prime}\right)$ and permeability $\left(\mu^{\prime}, \mu^{\prime \prime}\right)$ from the scattering parameters with the help of Nicolson- Ross- Weir (NRW) method (Paula, et al., 2011). Figure 4(a) \& 4(b) show the real and imaginary parts of complex relative permittivity spectra respectively. Figure 4(c) \& 4(d) show the real and imaginary parts of complex relative permeability. The real part of permittivity ( $\mathrm{e}^{\prime}$ ) is a measure of how much energy from an external electric field is stored in a material. The imaginary part of permittivity $\left(\mathrm{e}^{\prime \prime}\right)$ is known as loss factor and is a measure of how much energy dissipated or lost. The values of real parts of permittivity (e') for Graphene and MWCNT lie from 18.97 to 19.89 and 14.95 to 15.78 respectively. The values of imaginary parts of permittivity ( $\mathrm{e}^{\prime \prime}$ ) for Graphene and MWCNT lie from 9.40 to 8.26 and 2.90 to 3.8 respectively.According to Ref. (Watts, et al., 2003), there are many factors making contributions to the dielectric properties: dielectric relaxation, resonance, the motion of conduction electrons, defects in the nanotubes, length, diameters, chirality, etc. Therefore, it will be extremely difficult to describe the permittivity dispersion behaviors of Graphene/MWCNTs when taking into account of all these factors. Here, only two mechanisms may make major contributions to the permittivity dispersion of Graphene/MWCNT. One is the general permittivity behaviors of dielectric materials which can be described by the Cole-Cole model (Cole, et al., 1941; Li, 1990), such as electrons polarization, charges polarization, etc. The other one is due to the motion of conducting electrons (Leon, et al., 2001; Wu, et al., 2004; Schultz, et al., 2003). For instance, the contribution to the permittivity dispersion due to the conductivities has also been considered for Graphene/MWCNT composites in Ref. (Leon, et al., 2001) and Ref. (Wu, et al., 2004). From Figure 4(c), the value of real part of permeability ( $\mu^{\prime}$ ) for Graphene and MWCNT in TPU lie in the range from 1.10 to 0.89 and 1.08 to 0.88 respectively i.e. almost same for both the material. The imaginary part $\left(\mu^{\prime \prime}\right)$ is also equal for both in the frequency range $10-12 \mathrm{GHz}$ but in the lower region $(8-10 \mathrm{GHz})$ it is slightly different. The major portion of the plot lie from zero to negative region which means radiation passes through it without any absorption. But both the material showed a peak around $9.5 \mathrm{GHz}$ and the value is larger in case of Graphene.Graphene/MWCNT may show a little bit magnetic behavior due to the presence of some metals (Ni, Co, Fe etc.) as impurity (Han, et al., 2011). In spite of that there is a lot of report confirming the magnetic property of Graphene/MWCNT (Castro, et al., 2008; Ma, et al., 2004). The magnetic properties of Graphene depend upon many factors like the number of layers, the sample area (Ramakrishna, Matte, et al., 2009), crystallographic nature of Graphene and the nature of the edges etc. (Bhowmick, et al., 2008; Barone, et al., 2006; Son, et al., 2006). Magnetism of carbon material may also come due to the Adatom interstitial-vacancy (Ma, et al., 2004). Hence from the above discussion it can be concluded that Graphene/MWCNT has some sort of magnetic property.

\section{Conclusion}

In conclusion, Graphene and MWCNT both can be used as a RADAR absorbing material but Graphene is much superior to MWCNT. Here two different composite was prepared and the formation of composites with TPU was 
confirmed by FESEM and TEM analysis. The microwave absorption property was studied in detail and explained by the study of permittivity and permeability nature of the material. From the discussion it is cleared that dielectric loss is the most important contributing part than the magnetic loss. Though the value is very small but it is proved from their permeability plot that Graphene/MWCNT both has the magnetic property also. Hence Graphene/MWCNT can be used for the synthesis of different composite materials for this particular application. Our group is now working on the development of Graphene/MWCNT based composite materials which has a potential application as microwave absorbing material particularly in the X-band regionand in other region of the microwave too.

\section{Acknowledgements}

Thanks are due to CSIR, New Delhi, INDIA for the financial support in order to carry out this work.

\section{References}

Al-Saleh, M. H., \& Sundararaj, U. (2009). Electromagnetic interference shielding mechanisms of CNT/polymer composites. Carbon, 47, 1738-1746. http://dx.doi.org/10.1016/j.carbon.2009.02.030

Bhowmick, S., \& Shenoy, V. B. J. (2008). Edge State Magnetism of Single Layer Graphene Nanostructures. The Journal of Chemical Physics, 128, 244717. http://dx.doi.org/10.1063/1.2943678

Barone, V., Hod, O., \& Scuseria, G. E. (2006). Electronic Structure and Stability of Semiconducting Graphene Nanoribbons. Nano Letter. 6, 2748-2754. http://dx.doi.org/10.1021/n10617033

Castro, E. V., Peres, N. M. R., Stauber, T., \& Silva, N. A. P. (2008). Low-Density Ferromagnetism in Biased Bilayer Graphene. Physics Review Letter, 100, 186803. http://dx.doi.org/10.1103/PhysRevLett.100.186803.

Cole, K. S., \& Cole, R. H. (1941). Dispersion and Absorption in Dielectrics. Journal of Chemical Physics, 9 , 341-343. http://dx.doi.org/10.1063/1.1750906

Das, C. K., \& Mandal, A. (2011). Microwave Absorbing Properties of DBSA-doped Polyaniline $/ \mathrm{BaTiO}_{3}-\mathrm{Ni}_{0.5} \mathrm{Zn}_{0.5} \mathrm{Fe}_{2} \mathrm{O}_{4}$ Nanocomposites. Journal of Materials Science Research, 1(1), 45-53. http://dx.doi.org/10.5539/jmsr.v1n1p45

Fan, Z., Luo, G., Zhang, Z., Li Zhou, \& Fei. (2006). Electromagnetic and microwave absorbing properties of multi-walled carbon nanotubes/polymer composites. Materials Science and Engineering B, 132, 85-89.http://dx.doi.org/10.1016/j.mseb.2006.02.045

Gupta, A., \& Choudhary, V. (2011). Electromagnetic interference shielding behavior of poly (trimethylene terephthalate)/multi-walled carbon nanotube composites. Composites Science and Technology, 71, 1563-1568. http://dx.doi.org/10.1016/j.compscitech.2011.06.014

Han, M., \& Deng, L. (2011). High Frequency Properties of Carbon Nanotubes and Their Electromagnetic Wave Absorption Properties, Carbon Nanotubes Applications on Electron Devices. Jose Mauricio Marulanda (Ed.). ISBN: 978-953-307-496-2.

Hao, R., Qian, W., Zhang, L. H., \& Hou, Y. L. (2008). Aqueous dispersions of TCNQ-anion-stabilized Graphene sheets. Chemical Communication, 48, 6576-6578. http://dx.doi.org/10.1039/B816971C

Leon, C., Rivera, A., Varez, A., Sanz, J., \& Santamaria, J. (2001).Origin of constant loss in ionic conductors. Physical Review Letter, 86, 1279-1281. http://link.aps.org/doi/10.1103/PhysRevLett.86.1279

Li, N., Huang, Y., Du, F., He, X., Lin, X., \& Gao, H. (2006). Electromagnetic Interference (EMI) Shielding of Single-Walled Carbon Nanotube Epoxy Composites. Nano Letters, 6, 1141-1145.http://pubs.acs.org/doi/abs/10.1021/n10602589

Liu, Z., Bai, G., Huang, Y., Ma, Y., Du, F., Li, F., Guo, T., \& Chen, Y. (2007). Reflection and absorption contributions to the electromagnetic interference shielding of single-walled carbon nanotube/polyurethane composites. Carbon, 45, 821-827. http://dx.doi.org/10.1016/j.carbon.2006.11.020

Ma, C. C. M., Huang, Y. L., Kuan, H. C., \& Chiu, Y. S. (2005). Preparation and electromagnetic interference shielding characteristics of novel carbon-nanotube/siloxane/poly-(urea urethane) nanocomposites. Journal of Polymer Science Part B: Polymer Physics, 43, 345-358. http://dx.doi.org/10.1002/polb.20330

Matzui, L., Vovchenko, L., Prylutskyy, Yu., Korotash, I., Matzui, V., Eklund, P., Ritter, U., \& Scharff, P. (2007). Electromagnetic losses in carbon-epoxy composites. Materials Science and Engineering: C, 27, 1007-1009. http://dx.doi.org/10.1016/j.msec.2006.06.017 
Michielssen, E., Sajer, J., Ranjithan, S., \& Mittra, R. (1993). Design of Lightweight, Broad-Band Microwave Absorbers Using Genetic Algorithms. IEEE Trans. Microwave Theory Tech, 41, 1024-1030.

Paula, A. L., Rezende, M. C., \& Barroso, J. J. (2011). Experimental measurements and numerical simulation of permittivity and permeability of Teflon in X band. Journal of Aerospace Technology and Management, 3, 59-64. http://dx.doi.org/10.5028/jatm.2011.03019410

Ramakrishna Matte, H. S. S., Subrahmanyam, K. S., \& Rao, C. N. R. (2009). Novel Magnetic Properties of Graphene: Presence of both Ferromagnetic and Antiferromagnetic Features and Other Aspects. Journal of Physical Chemistry C, 113, 9982-9985. http://dx.doi.org/10.1021/jp903397u

Schultz, J. W., \& Moore, R. L. (2003). Effective Medium Calculations of the Electromagnetic Behavior of Single Walled Carbon Nanotube Composites. Material Research Society Symposium Proceedings, 739, 151-153. http://dx.doi.org/10.1557/PROC-739-H7.42

Son, Y. W., Cohen, M. L., \& Louie, S. G. (2006). Half-metallic Graphene Nanoribbons. Nature, 444, 347-349. http://dx.doi.org/10.1038/nature05180

Wang, Y., \& Jing, X. (2005). Intrinsicallyconducting polymers for electromagnetic interference shielding. Polymers for Advanced Technologies, 16, 344-351. http://onlinelibrary.wiley.com/doi/10.1002/pat.589/abstract

Watts, P. C. P., Ponnampalam, D. R., Hsu, W. K., Barnes, A., \& Chambers, B. (2003).The complex permittivity of multi-walled carbon nanotube-polystyrene composite films in X-band. Chemical Physics Letters, 378, 609-614. http://dx.doi.org/10.1016/j.cplett.2003.07.002

Watts, P. C. P., Hsu, W., \& Barnes, K. (2003). A high permittivity from defective multiwalled carbon nanotubes in the X-Band. Advanced Materials, 15, 600-603. http://dx.doi.org/10.1002/adma.200304485

Wu, J. H., \& Kong, L. B. (2004). High microwave permittivity of multiwalled carbon nanotube composites. Applied Physics Letter, 84, 49561-49563. http://dx.doi.org/10.1063/1.1762693

Yuchen M., Lehtinen,P. O.,Foster,A. S., \& Nieminen, R. M. (2004). Magnetic properties of vacancies in Graphene and single-walled carbon nanotubes. New Journal of Physics, 6, 68. http://dx.doi.org/10.1088/1367-2630/6/1/068.
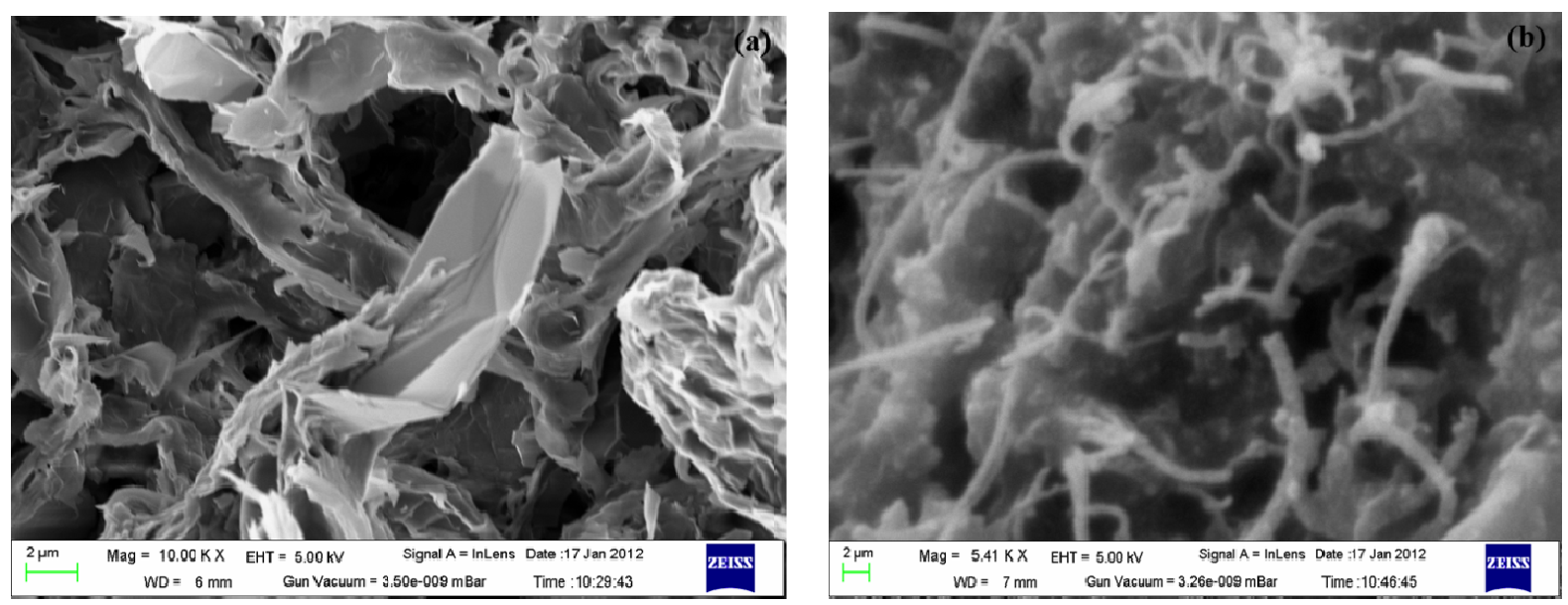

Figure 1. FESEM images of (a) Graphene and (b) MWCNT in TPU 

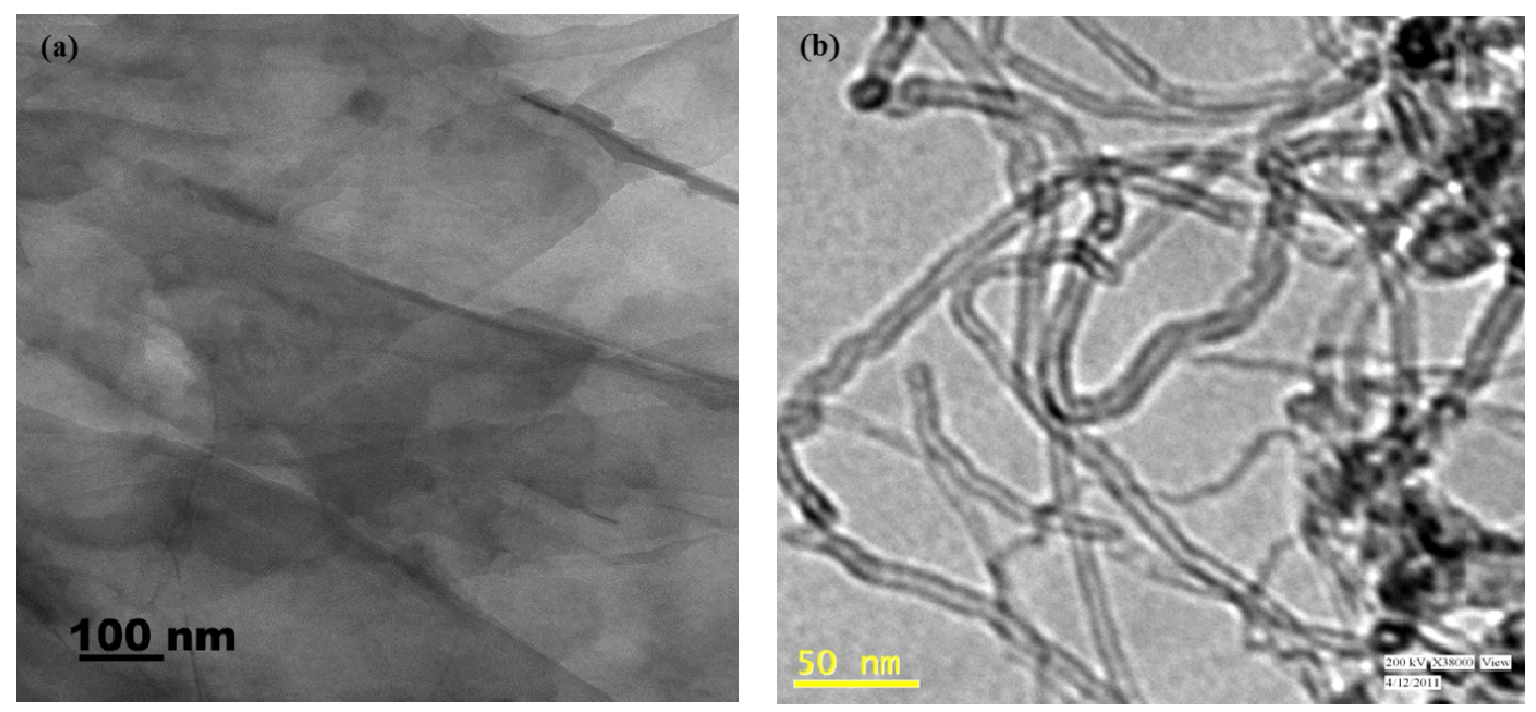

Figure 2. TEM images of (a) Graphene and (b) MWCNT in TPU

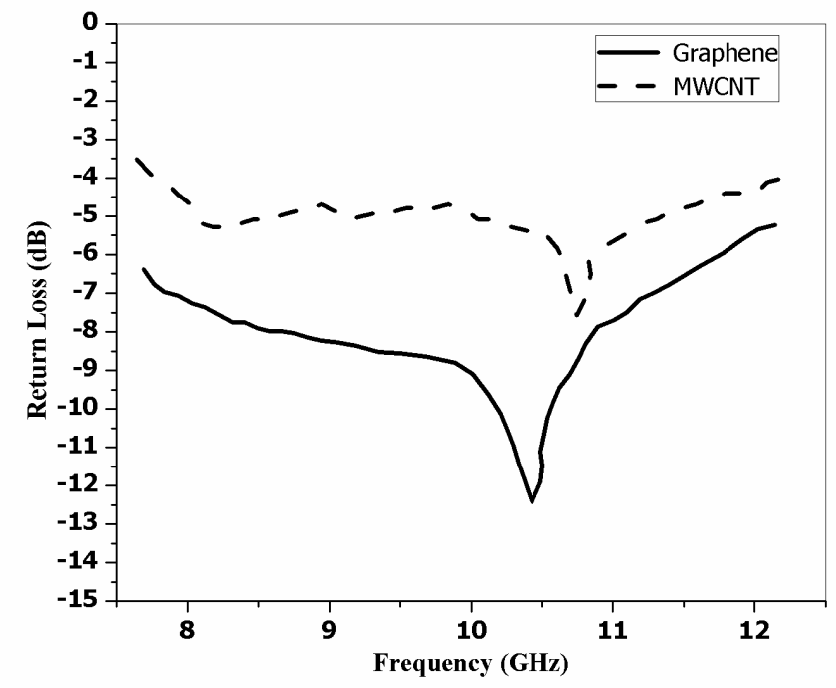

Figure 3. Return loss Vs frequency of Graphene and MWCNT 

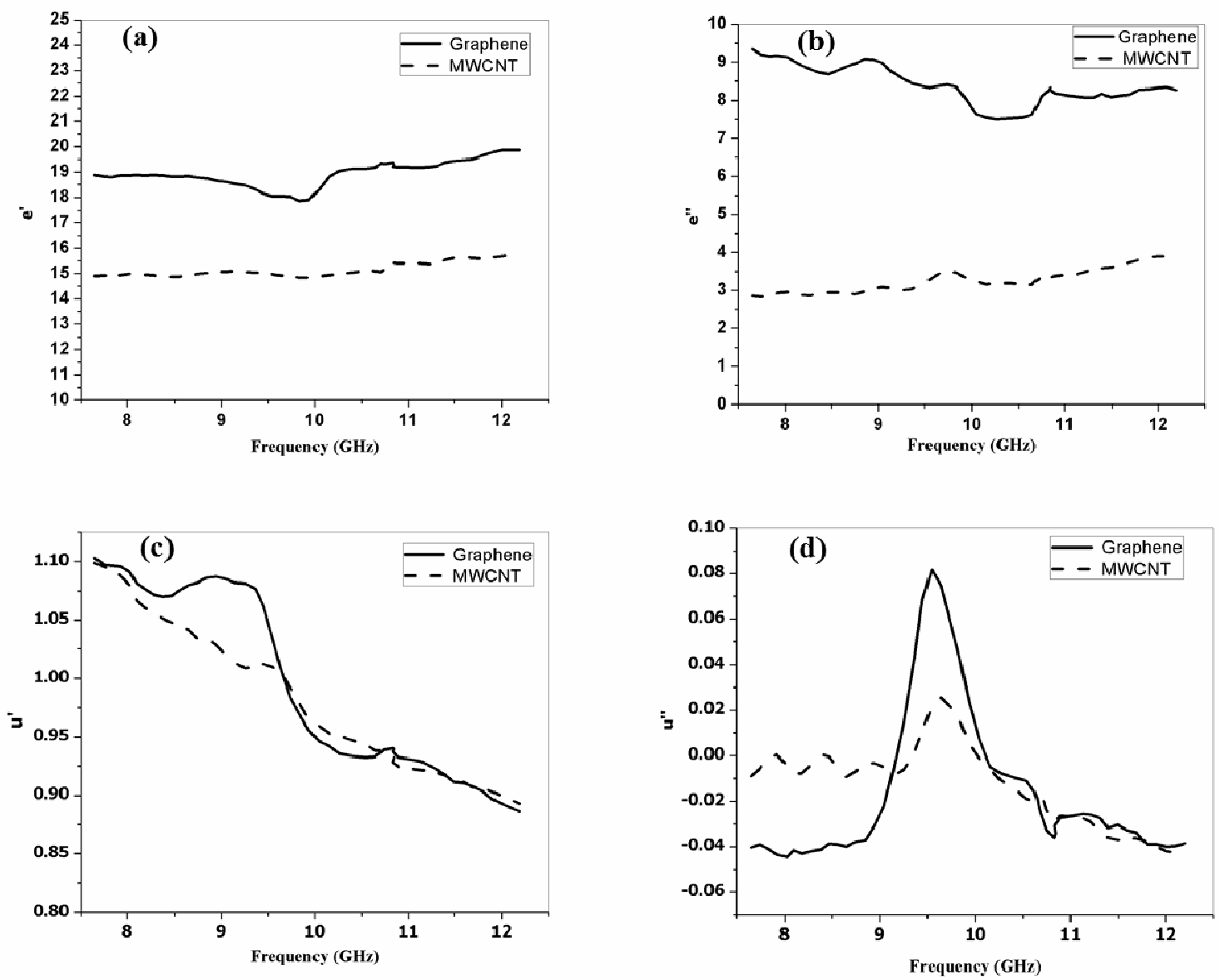

Figure 4. (a) Real (e') and (b) imaginary (e's) parts of relative complex permittivity, (c) real ( $\left.\mu^{\prime}\right)$ and (d) imaginary $\left(\mu^{\prime \prime}\right)$ parts of relative complex permeability of prepared RAMs 